chromosphere and which ultimately derive their energy from the accretion of hot gas by the Galaxy. Whether the flux of ionizing photons is sufficient depends on its unknown spectrum, but in principle there would be enough flux available.

A further possibility is that sufficient photons may enter the disk of the Galaxy to contribute appreciably to the overall ionization rate of the interstellar medium. We know from the work of Bergeron and Souffrin ${ }^{15}$ and of Habing and Goldsmith $^{16}$ that if the disk is ionized by $\sim 100 \mathrm{eV}$ photons then we would need a primary hydrogen ionization rate $\sim 10^{-16} \mathrm{~s}^{-1}$ to account for the observed ionization state and temperature of the interstellar medium. The actual ionization mechanism is still not known. A likely possibility is radiation from the discrete galactic soft X-ray sources discussed by Gorenstein and Tucker ${ }^{11}$ and by Davidsen et al. ${ }^{17}$. However, radiation from the galactic chromosphere is an alternative possibility which might be important close to the edge of the disk.

As a final speculative remark I would mention the possibility that it is the inflowing material which is responsible for the confinement of the interstellar magnetic field and cosmic rays to the disk of the Galaxy. This is dynamically reasonable since with our accretion parameters the internal and ram pressures of the incoming material $\sim 10^{-12}$ dyne $\mathrm{cm}^{-2}$ near the disk. I hope to return to this dynamical question elsewhere.

I thank R. Hunt, N. L. Balazs, A. Gabriel and M. S. Longair for helpful discussions and M. J. Rees for valuable criticisms of the first version of this letter.

Department of Astrophysics,

D. W. SCIAMA

Oxford University

Received August 17; revised November 7, 1972.

1 Spitzer, L., Astrophys. J., 124, 20 (1956).

2 Sciama, D. W., Mon. Not. Roy. Astron. Soc., 123, 317 (1962).

3 Hunt, R., and Sciama, D. W., Mon. Not. Roy. Astron. Soc., 157, 335 (1972).

4 Hunt, R., Mon. Not. Roy. Astron. Soc., 154, 141 (1971).

5 Salpeter, E. E., Interstellar Gas Dynamics, IAU Symposium No. 39, 221 (Reidel, Dordrecht, 1970).

6 Tarter, C. B., and Salpeter, E. E. Astrophys. J., 156, 943 (1969).

7 Tarter, C. B., Tucker, W. H., and Salpeter, E. E., Astrophys. J., 156, 953 (1969)

8 Cox, D. P., and Tucker, W. H., Astrophys. J., 157, 1157 (1969).

9 Purton, C. R., thesis, University of Cambridge (1966).

10 Bridle, A. H., and Venugopal, V. R., Nature, 224, 545 (1969).

11 Gorenstein, P., and Tucker, W. H., Astrophys. J., 176, 333 (1972).

12 Hunt, R., and Sciama, D. W., Nature, 238, 320 (1972).

13 Felten, J. E., and Bergeron, J., Astrophys. Lett., 4, 155 (1969).

${ }^{14}$ Silk, J., Astron. Astrophys., 12, 421 (1971).

15 Bergeron, J., and Souffrin, S., Astron. Astrophys., 11, 40 (1971).

16 Habing, H. J., and Goldsmith, D. W., Astrophys. J., 166, 525 (1971).

${ }^{17}$ Davidsen, A., Shulman, S., Fritz, G., Meekins, J. F., Henry, R. C., and Friedman, H., Astrophys. J. (in the press).

\section{Is There a Tenth Planet in the Solar System?}

WE have been analysing the longitudinal residuals of Neptune $(\mathrm{N})$, partly from sheer interest aroused by the discordance between the pre-discovery position of $\mathrm{N}$ and its currently accepted orbit ${ }^{1}$ and because colleagues (already conducting a photographic hunt for a hypothetical tenth planet) sought our assistance in narrowing the field of search by using perturbation theory.

Seidelmann $^{2}$ has ruled out any of three possible outer planets-and Brady's suggestion ${ }^{3}$ of a planet of Jovian mass at about $60 \mathrm{AU}$ seems hardly possible, because its effect on Uranus (U) and N (easily computed from ref. 4, Fig. 4) would be larger by orders of magnitude than any residual observed since the mid nineteenth century. Further, a search by Foss et al. has failed to find $\mathrm{it}^{5}$. We therefore decided to attempt to establish the limits of the possible position of a tenth planet, working from data on $\mathrm{N}$.

$\mathrm{N}$ was chosen as the main basis for the work because it is the most sensitive of the known planets to the pull of a possible exterior body ${ }^{4}$ and the least affected by existing uncertainties in the masses of its known planetary neighbours. As a check on the working, data from Uranus (U) were also used; and cases where the results from these two sources were markedly different were rejected.

A least-squares differential analysis was carried out, assuming circular orbits for any perturbing body. This assumption was made because it is reasonable a priori, and because it was decided that the $\mathrm{N}$ data were insufficient to indicate reliably the extra unknowns involved in an eliptical path. (A paper giving full details of the work is in preparation.)

The only satisfactory solutions found were for bodies whose orbits lay between 50 and $100 \mathrm{AU}$ from the Sun, and whose positions at 1973.0 lay within a relatively small sector of the ecliptic. The range of such possible bodies is shown in Table 1.

Table 1 Limits of Solutions for a Possible Tenth Planet

$\begin{array}{ccc}\text { Radius of orbit (AU) } & \text { Longitude limits (2 s.d.) } & \text { Mass } \pm 1 \text { s.d. } \\ 75 & 309^{\circ}-320^{\circ} & 5 \pm 2 \\ 60 & 322^{\circ}-343^{\circ} & 3 \pm 1 \\ 50 & 338^{\circ}-5^{\circ} & 2 \pm 1\end{array}$

Masses are in Sun/206265.

(Epoch: 1970.0; E and E 1950.0.)

These results and N's latitude residuals were used to find latitude solutions. Unfortunately, however, the standard deviations of the latitude results are large, and the confidence intervals correspondingly so (Table 2 ).

Table 2 Latitude Solutions for Various Radius and Longitude Values

\begin{tabular}{ccc}
\hline Radius of orbit (AU) & Longitude & Latitude \pm 1 s.d. \\
75 & $310^{\circ}$ & $-17^{\circ} \pm 37^{\circ}$ \\
& $320^{\circ}$ & $-27^{\circ} \pm 40^{\circ}$ \\
60 & $320^{\circ}$ & $+6^{\circ} \pm 8^{\circ}$ \\
& $340^{\circ}$ & $+30^{\circ} \pm 17^{\circ}$ \\
50 & $350^{\circ}$ & $+14^{\circ} \pm 6^{\circ}$ \\
& $0^{\circ}$ & $+20^{\circ} \pm 10^{\circ}$ \\
\hline
\end{tabular}

Seidelmann ${ }^{2}$ rejected 3 trial planets, as the only one which accounted for the 1795 residual forced unacceptably large ones upon N's motion since 1846. A body falling within our range, however, satisfies the former whilst only requiring disturbances of amplitude ca. $0.05 \mathrm{~s}$ in the post-1846 data.

Nevertheless we do not definitely assert that such a planet exists. Our set task, which we consider we have performed, was to delimit the possible space wherein it might be.

If the current search in this area finds nothing, then the indication becomes strong that the major bodies of the solar system end at $\mathrm{N}$, a matter bearing critically upon theories of the origin of the solar system.

We thank Dr D. Barton, Dr J. Rozics, Mr J. Lang, Miss E. Sadie and Miss A. Avirett for their assistance in the work and also Dr D. W. Dewhirst for help and advice.

College of Notre Dame of Maryland, Baltimore

D. RAWLINS

M. HAMMERTON

\section{APU Cambridge, England}

Received October 17, 1972.

1 Rawlins, D., Astrophys. J., 75, 856 (1970).

2 Seidelmann, P., Astrophys. J., 76, 740 (1971).

3 Brady, J., Publ. Astr. Soc. Pacific, 84, 314 (1972).

4 Rawlins, D., Mon. Not. Roy. Astron. Soc., 147, 177 (1970).

5 Foss, A., Shawe-Taylor, J., and Whitworth, D., Nature, 266, 239 (1972). 